\title{
A jet proper motion study in the early Universe
}

\author{
Krisztina Perger ${ }^{1,2}$, Sándor Frey ${ }^{2}$ and Krisztina É. Gabányi ${ }^{2,3}$ \\ ${ }^{1}$ Department of Astronomy, Eötvös Loránd University \\ Pázmány Péter sétány 1/A, H-1117 Budapest, Hungary \\ email: k.perger@astro.elte.hu \\ ${ }^{2}$ Konkoly Observatory, MTA Research Centre for Astronomy and Earth Sciences \\ Konkoly Thege Miklós út 15-17, H-1121 Budapest, Hungary \\ ${ }^{3}$ MTA-ELTE Extragalactic Astrophysics Research Group \\ Pázmány Péter sétány 1/A, H-1117 Budapest, Hungary
}

\begin{abstract}
We constrained the jet proper motion of PMN J2134-0419 at $z=4.33$ to $\mu=0.035 \pm$ $0.023 \mathrm{mas} \mathrm{yr}^{-1}$ using very long baseline interferometry (VLBI). This is the second most distant source where such a measurement could be made. Another distant blazar SDSS J1026+2542

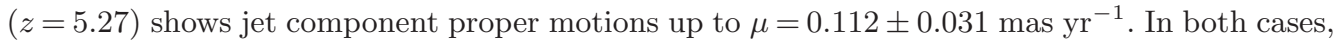
the measured values are consistent with the expectations of current $\Lambda$ CDM cosmological models.
\end{abstract}

Keywords. galaxies: active, galaxies: jets, galaxies: high-redshift, quasars: individual (PMN J2134-0419), quasars: individual (SDSS J1026+2542)

\section{Introduction}

Observing high-redshift active galactic nuclei (AGN) at radio wavelengths provides crucial information about the early epochs of our Universe. Using the very long baseline interferometry (VLBI) technique, the structure of radio jets can be studied with milliarcsecond (mas) resolution at $\mathrm{cm}$ wavelengths. With observations at two suitably distant epochs, the proper motion of the jet components and the physical and geometric properties of the plasma flow can be determined.

So far there are only two AGN at the highest redshifts (PMN J2134-0419 at $z=4.33$ and SDSS J1026+2542 at $z=5.27$ ) that show suitably extended mas-scale jet structure and have VLBI imaging data at two epochs so that a jet proper motion study could be performed (Frey et al., 2015; Perger et al., 2018).

\section{Radio jet proper motion at $z>4$}

J1026+2542 was observed with the Very Long Baseline Array (VLBA) and with the European VLBI Network (EVN), in 2006 and 2013, respectively, while J2134-0419 was observed by the EVN in 1999 and 2015. All observations were carried out at $5 \mathrm{GHz}$.

The two-epoch analysis of the radio emission in the parsec-scale jet of both AGN indicates an outward motion. J1026+2542 has multiple jet components, while J21340419 exhibits a more simple structure. The change in the angular distance of the core

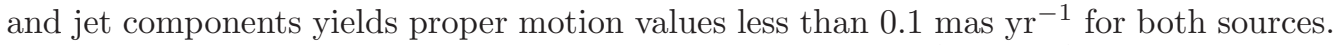

Applying standard $\Lambda$ CDM cosmology $\left(H_{0}=70 \mathrm{~km} \mathrm{~s}^{-1} \mathrm{Mpc}^{-1}, \Omega_{\mathrm{M}}=0.3\right.$, and $\left.\Omega_{\Lambda}=0.7\right)$, the proper motions can be translated to apparent superluminal speeds, which span the range of $[3.3 \pm 3.9,14.0 \pm 3.9] c$ for $\mathrm{J} 1026+2542$, and has the value of $4.1 \pm 2.7 c$-for J2134-0419. The calculated values of the bulk Lorentz factor and the jet 


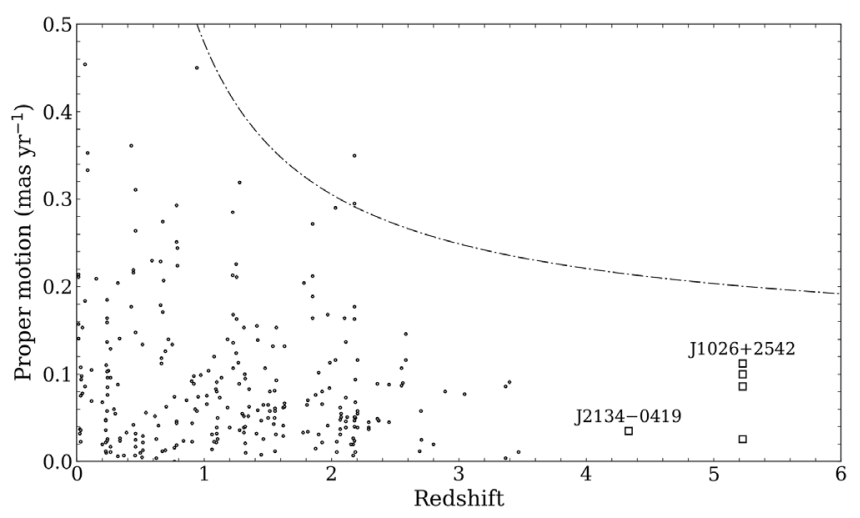

Figure 1. Distribution of jet proper motions in AGN as a function of redshift. Circles denote the best quality flag sources from Britzen et al. (2008) for comparison at lower redshifts. The curve represents the upper limit based on the applied cosmology, assuming a Lorentz-factor $\Gamma=25$.

inclination angle to the line of sight span the ranges $\Gamma=12.5-15$ and $\theta=4.6^{\circ}-10^{\circ}$ for $\mathrm{J} 1026+2542$, and the ranges $\Gamma=2-7$ and $\theta=3^{\circ}-20^{\circ}$ for $\mathrm{J} 2134-0419$.

\section{Conclusions}

The constraints obtained on the jet proper motion of the two high-redshift AGN are consistent with the upper limit estimated by the current cosmological models (e.g. Kellermann et al. 2004). The determined Lorentz factor and viewing angle are consistent with those obtained from models fitted to the broad-band spectral energy distribution (SED, Sbarrato et al. 2013) for J1026+2542. The radio interferometric data show slightly lower Lorentz factor and higher viewing angle for J2134-0419 than the SED study (Sbarrato et al. 2015).

\section{Acknowledgements}

$\mathrm{KP}$ is grateful for the IAU grant supporting her to attend the Symposium. KÉG was supported by the János Bolyai Research Scholarship of the Hungarian Academy of Sciences. We thank the Hungarian National Research, Development and Innovation Office (OTKA NN110333) for support.

\section{References}

Britzen, S., Vermeulen, R. C., Campbell, R. M., et al. 2008, A\& A, 484, 119

Frey, S., Paragi, Z., Fogasy, J. O. \& Gurvits, L. I. 2015, MNRAS, 446, 2921

Kellermann K. I., Lister, M. L., Homan, D. C. et al. 2004, ApJ, 609, 539

Perger, K., Frey, S., Gabányi, K. É. et al. 2018, MNRAS 477, 1065

Sbarrato, T., Tagliaferri, G., Ghisellini, G. et al. 2013, ApJ, 777, 147

Sbarrato, T., Ghisellini, G., Tagliaferri, G. et al. 2015 MNRAS, 446, 2483 